\title{
Generalized Fibonacci - Like Sequence Associated with Fibonacci and Lucas Sequences
}

\author{
Yogesh Kumar Gupta ${ }^{1, *}$, Mamta Singh $^{2}$, Omprakash Sikhwal $^{3}$ \\ ${ }^{1}$ Schools of Studies in Mathematics, Vikram University Ujjain, (M. P.) India \\ ${ }^{2}$ Department of Mathematical Sciences and Computer application, Bundelkhand University, Jhansi (U. P.) \\ ${ }^{3}$ Department of Mathematics, Mandsaur Institute of Technology, Mandsaur (M. P.) India \\ *Corresponding author: yogeshgupta.880@rediffmail.com
}

Received November 12, 2014; Revised December 20, 2014; Accepted December 28, 2014

\begin{abstract}
The Fibonacci sequence, Lucas numbers and their generalization have many interesting properties and applications to almost every field. Fibonacci sequence is defined by the recurrence formula $F_{n}=F_{n-1}+F_{n-2}, n \geq 2$ and $F_{0}=0, F_{1}=1$, where $F_{n}$ is a $n^{\text {th }}$ number of sequence. Many authors have been defined Fibonacci pattern based sequences which are popularized and known as Fibonacci-Like sequences. In this paper, Generalized Fibonacci-Like sequence is introduced and defined by the recurrence relation $B_{n}=B_{n-1}+B_{n-2}, n \geq 2$ with $\mathrm{B}_{0}=2 s, \mathrm{~B}_{1}=s+1$, where $s$ being a fixed integers. Some identities of Generalized Fibonacci-Like sequence associated with Fibonacci and Lucas sequences are presented by Binet's formula. Also some determinant identities are discussed.
\end{abstract}

Keywords: Fibonacci sequence, Lucas sequence, Generalized Fibonacci-Like Sequence, Binet's formula

Cite This Article: Yogesh Kumar Gupta, Mamta Singh, and Omprakash Sikhwal, "Generalized Fibonacci Like Sequence Associated with Fibonacci and Lucas Sequences." Turkish Journal of Analysis and Number Theory, vol. 2, no. 6 (2014): 233-238. doi: 10.12691/tjant-2-6-9.

\section{Introduction}

The Fibonacci and Lucas sequences are well-known examples of second order recurrence sequences. The Fibonacci numbers are perhaps most famous for appearing in the rabbit breeding problem, introduced by Leonardo de Pisa in 1202 in his book called Liber Abaci. As illustrate in the tome by Koshy [15] the Fibonacci and Lucas number are arguable two of the most interesting sequence in all of mathematics. Many identities have been documented in an extensive list that appears in the work of Vajda [14], where they are proved by algebra means, even though combinatorial proof of many of these interesting identities. We introduced Generalized Fibonacci-Like Sequence and some identities Fibonacci numbers, Lucas number's and their generalization have many interesting Properties and application to almost every field.

The Fibonacci sequence [5] is a sequence of numbers starting with integer 0 and 1 , where each next term of the sequence calculated as the sum of the previous two.

$$
\begin{aligned}
& \text { i.e., } F_{n}=F_{n-1}+F_{n-2}, n \geq 2 \\
& \text { and } F_{0}=0, F_{1}=1 \text {. }
\end{aligned}
$$

The similar interpretation also exists for Lucas sequence. Lucas sequence [10] is defined by the recurrence relation,

$$
\begin{aligned}
& \mathrm{L}_{\mathrm{n}}=\mathrm{L}_{\mathrm{n}-1}+\mathrm{L}_{\mathrm{n}-2}, \mathrm{n} \geq 2 \\
& \text { and } \mathrm{L}_{0}=2, \mathrm{~L}_{1}=1
\end{aligned}
$$

In this paper, we present various properties of the Generalized Fibonacci-Like sequence (GFLS) associated with Fibonacci and Lucas sequences $\left\{B_{n}\right\}$ defined by

$$
B_{n}=B_{n-1}+B_{n-2}, n \geq 2 \text { and } \mathrm{B}_{0}=2 s, \mathrm{~B}_{1}=s+1 \text {. }
$$

The Binet's formula for Fibonacci sequence is given by

$$
F_{n}=\frac{\Re_{1}^{n}-\Re_{2}^{n}}{\mathfrak{R}_{1}-\mathfrak{R}_{2}}=\frac{1}{\sqrt{5}}\left\{\left(\frac{1+\sqrt{5}}{2}\right)^{n}-\left(\frac{1+\sqrt{5}}{2}\right)^{n}\right\}
$$

where $\mathfrak{R}_{2}=\frac{1+\sqrt{5}}{2}$ Golden ratio $=1.618$

and $\Re_{2}=\frac{1-\sqrt{5}}{2}$ Golden ratio $=-0.618$

Similarly, the Binet's formula for Lucas sequence is given by

$$
L_{n}=\Re_{1}^{n}+\Re_{2}^{n}=\left\{\left(\frac{1+\sqrt{5}}{2}\right)^{n}-\left(\frac{1+\sqrt{5}}{2}\right)^{n}\right\} .
$$

\section{Preliminary Results Generalized Fibonacci-Like Sequence}

We need to introduce some basic results of Generalized Fibonacci-Like sequence associated with Fibonacci and Lucas sequences $\left\{\mathrm{B}_{\mathrm{n}}\right\}$ is defined by recurrence relation: 


$$
B_{n}=B_{n-1}+B_{n-2}, \mathrm{n} \geq 2
$$

With initial conditions $\mathrm{B}_{0}=2 \mathrm{~s}$ and $\mathrm{B}_{1}=\mathrm{s}+1$.

The associated initial Condition $\mathrm{B}_{0}$ and $\mathrm{B}_{1}$ are the sum of initial condition of generalized Fibonacci-Like sequence respectively.

$$
\text { i.e. } F_{0}+s_{0}=B_{0} \text { and } F_{1}+s_{1}=B_{1}
$$

The few terms of above sequence are $2 \mathrm{~s}, \mathrm{~s}+1,1+3 \mathrm{~s}$, $2+4 \mathrm{~s}, 3+7 \mathrm{~s}$, and so on.

The relation between Fibonacci sequence and Generalized Fibonacci-Like Sequence can be written as

$$
\mathrm{B}_{\mathrm{n}}=\mathrm{F}_{\mathrm{n}}+\mathrm{sL}_{\mathrm{n}}, n \geq 0 .
$$

The recurrence relation (1.1) has the characteristic equation $x^{2}=x+1$ which has two roots

$$
\mathfrak{R}_{1}=\frac{1+\sqrt{5}}{2} \text { and } \mathfrak{R}_{2}=\frac{1-\sqrt{5}}{2} \text {. }
$$

Now notice a few things about $\mathfrak{R}_{1}$ and $\mathfrak{R}_{2}$

$$
\begin{aligned}
& \mathfrak{R}_{1}+\mathfrak{R}_{2}=1, \mathfrak{R}_{1}-\mathfrak{R}_{2}=\sqrt{5} \\
& \text { and } \mathfrak{R}_{1} \mathfrak{R}_{2}=-1 \text {. }
\end{aligned}
$$

Using these two roots, we obtain Binet's recurrence relation

$$
\begin{aligned}
& B_{n}=\frac{\mathfrak{R}_{1}^{n}-\mathfrak{R}_{2}^{n}}{\sqrt{5}}+s\left(\mathfrak{R}_{1}^{n}+\mathfrak{R}_{2}^{n}\right) \\
& =\frac{1}{\sqrt{5}}\left\{\left(\frac{1+\sqrt{5}}{2}\right)^{n}-\left(\frac{1+\sqrt{5}}{2}\right)^{n}\right\} \\
& +s\left\{\left(\frac{1+\sqrt{5}}{2}\right)^{n}+\left(\frac{1-\sqrt{5}}{2}\right)^{n}\right\}
\end{aligned}
$$

\section{Generating Function}

Now we state derive generating function of generalized Fibonacci-Like sequence

$$
\sum_{n=0}^{\infty} B_{n} x^{n}=\frac{2 s+(1-s) x}{\left(1-x-x^{2}\right)}
$$

Let's apply power series to sequence $\left\{B_{n}\right\}$

Let $2 \mathrm{~s}+(\mathrm{s}+1) \mathrm{x}+(1+3 \mathrm{~s}) \mathrm{x}^{2}+\ldots \ldots \ldots \ldots . . . .=\sum_{n=0}^{\infty} B_{n} x^{n}$

Where $\mathrm{B}_{\mathrm{n}}$ is $\mathrm{n}^{\text {th }}$ term of sequence $\left\{B_{n}\right\}$.

This is called generating series of Generalized Fibonacci - Like Sequence $\left\{B_{n}\right\}$.

Now multiplying the generating series

$$
\begin{aligned}
& \left(1-x-x^{2}\right) \sum_{n=0}^{\infty} B_{n} x^{n} \\
& =\sum_{n=0}^{\infty} B_{n} x^{n}-\sum_{n=0}^{\infty} B_{n} x^{n+1}-\sum_{n=0}^{\infty} B_{n} x^{n+2}
\end{aligned}
$$

$$
\begin{aligned}
& =\left(B_{0}+B_{1} x+\sum_{n=2}^{\infty} B_{n} x^{n}\right) \\
& -B_{0} x+\sum_{n=2}^{\infty} B_{n-1} x^{n}-\sum_{n=2}^{\infty} B_{n-2} x^{n} \\
& =B_{0}+\left(B_{1}-B_{0}\right) x+\sum_{n=2}^{\infty}\left(B_{n}-B_{n-1}-B_{n-2}\right) x^{n} \\
& =2 s+(s+1-2 s) x+\sum_{n=2}^{\infty}\left(\begin{array}{l}
B_{n-1}+B_{n-2} \\
-B_{n-1}-B_{n-2}
\end{array}\right) x^{n} \\
& =2 s+(s+1-2 s) x+\sum_{n=2}^{\infty}(0) x^{n} \\
& =2 s+(s+1-2 s) x
\end{aligned}
$$

Therefore, $\left(1-x-x^{2}\right) \sum_{n=0}^{\infty} B_{n} x^{n}=2 s+(1-s) x$.

Hence $\sum_{n=0}^{\infty} B_{n} x^{n}=\frac{2 s+(1-s) x}{\left(1-x-x^{2}\right)}$.

\section{Properties of Generalized Fibonacci- Like Sequence}

Despite its simple appearance the Generalized Fibonacci-Like sequence $\left\{\mathrm{B}_{\mathrm{n}}\right\}$ contains a wealth of subtle and fascinating properties $[4,6,9,12]$.

\section{Sum of n First terms:}

Theorem (4.1). Let $B_{n}$ be the $n^{\text {th }}$ Fibonacci-Like number, then Sum of the first $n$ terms of generalized FibonacciLike sequence is

$$
\left(B_{1}+B_{2}+B_{3}+\ldots \ldots B_{n}\right)=\sum_{k=1}^{n} B_{k}=B_{n+2}-(1+3 s)(4.1)
$$

Proof: we know that the follows relation holds:

$$
\begin{aligned}
& B_{1}=B_{3}-B_{2} \\
& B_{2}=B_{4}-B_{3} \quad\left(\text { Since } B_{3}=B_{1}+B_{2}\right) \\
& B_{3}=B_{5}-B_{4} \\
& -------- \\
& -------- \\
& B_{n-1}=B_{n+1}-B_{n} \\
& B_{n}=B_{n+2}-B_{n+1}
\end{aligned}
$$

Term wise addition of all above equations, we obtain

$$
\begin{aligned}
\left(B_{1}+B_{2}+B_{3}+\ldots \ldots B_{n}\right) & =B_{n+2}-B_{2} \\
& =B_{n+2}-(1+3 s)
\end{aligned}
$$

\section{Sum of First $n$ terms with even indices}

Theorem (4.2). Let $B_{n}$ be the $n^{\text {th }}$ Fibonacci-Like sequence, then Sum of the first $\mathrm{n}$ terms with even indices is

$$
\left(B_{2}+B_{4}+B_{6}+\ldots \ldots B_{2 n}\right)=\sum_{k=1}^{n} B_{2 k}=B_{2 n+1}-1+s(4.2)
$$


Sum of First $n$ terms with square indices:

Theorem (4.3). Let $B_{n}$ be the $n^{\text {th }}$ Fibonacci-Like sequence, then Sum of the square of first $n$ terms is

$$
\left(B_{1}^{2}+B_{2}^{2}+B_{3}^{2}+\ldots \ldots \ldots B_{n}^{2}\right)=\sum_{k=1}^{n} B_{k}^{2}=B_{n} B_{n-2}
$$

\section{Sum of First $n$ terms with odd indices:}

Theorem (4.4). Let $B_{n}$ be the $n^{\text {th }}$ Fibonacci-Like sequence, then Sum the first $\mathrm{n}$ terms with odd indices is

$$
\begin{aligned}
& \left(B_{1}+B_{3}+B_{5}+B_{7}+\ldots \ldots \ldots+B_{2 n-1}\right) \\
& =\sum_{k=1}^{n} B_{2 k-1}=B_{2 n}-B_{2 n-2}
\end{aligned}
$$

Now we state and prove some nice identities similar to those obtained for Fibonacci and Lucas sequences $[1,2,4,12]$.

\section{Some Identities Generalized Fibonacci- Like Sequence}

In this section, some identities of Generalized Fibonacci-Like sequence are presented which can be easily derived by Explicit sum formula using generating function and Binet's formula. Authors [5,6] have been described such type identities.

Explicit Sum Formula:

Theorem (5.1). The explicit sum formula for Generalized Fibonacci-Like sequence is given by For positive integer $n$, Prove that

$$
B_{2 n}=\sum_{m=0}^{n}\left(\begin{array}{c}
n \\
m
\end{array}\right) B_{n-m}
$$

Proof: By equation (2.1), it follows that

$$
\begin{aligned}
& B_{2 n}=B_{2 n-1}+B_{2 n-2} \\
& =\left(B_{2 n-2}+B_{2 n-3}\right)+\left(B_{2 n-3}+B_{2 n-4}\right) \\
& =B_{2 n-2}+2 B_{2 n-3}+B_{2 n-4} \\
& =\left(B_{2 n-3}+B_{2 n-4}\right)+2\left(B_{2 n-4}+B_{2 n-5}\right) \\
& +\left(B_{2 n-5}+B_{2 n-6}\right)
\end{aligned}
$$

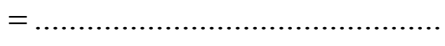

$$
\begin{aligned}
& = \\
& =B_{0}+n B_{1}+\frac{n(n-1)}{2} B_{2}+ \\
& +\frac{n(n-1)}{2} B_{n-2}+n B_{n-1}+B_{n} \\
& \text { Hence } \mathrm{B}_{2 \mathrm{n}}=\sum_{m=0}^{n}\left(\begin{array}{l}
n \\
m
\end{array}\right) B_{n-m} \text {. }
\end{aligned}
$$

Theorem (2). The explicit sum formula for Generalized Fibonacci-Like sequence is given by For positive integer n,

$$
B_{n}=\sum_{k=0}^{n}\left(\begin{array}{l}
n \\
k
\end{array}\right) B_{n-2 k}
$$

Theorem (5.3). For every positive integer n, prove that

$$
B_{m+1} B_{n}-B_{m+1} B_{n+1}=(-1)^{n} B_{m+1} B_{n-m+1}, n \geq 1
$$

Proof: Let $\mathrm{n}$ be fixed and we Proved by inducting on $\mathrm{m}$. When $\mathrm{m}=0$, then

$$
\begin{aligned}
& B_{1} B_{n}-B_{1} B_{n+1}=(-1)^{1} B_{1} B_{n-1} \\
& (1+s) B_{n}-(1+s) B_{n+1}=-(1+s) B_{n-1} \\
& (1+s)\left(B_{n}-B_{n+1}\right)=-(1+s) B_{n-1} \\
& (1+s)\left(-B_{n-1}\right)=-(1+s) B_{n-1} \\
& -(1+s) B_{n-1}=-(1+s) B_{n-1}
\end{aligned}
$$

Which is true.

When $\mathrm{m}=1$, then

$$
\begin{aligned}
& B_{1+1} B_{n}-B_{1+1} B_{n+1}=(-1)^{1} B_{1+1} B_{n-1-1} \\
& B_{2} B_{n}-B_{2} B_{n+1}=(-1)^{1} B_{2} B_{n-2} \\
& B_{2}\left(B_{n}-B_{n+1}\right)=(-1)^{1} B_{2} B_{n-2} \\
& (1+3 s)\left(B_{n}-B_{n+1}\right)=(-1)^{1}(1+3 s) B_{n-2} \\
& (1+3 s)\left(-B_{n-2}\right)=-(1+3 s) B_{n-2} \\
& -(1+3 s) B_{n-2}=-(1+3 s) B_{n-2}
\end{aligned}
$$

which also is true.

Now assume that identity is true for $\mathrm{m}=\mathrm{k}+1$, then by assumption

$$
\begin{aligned}
B_{k} B_{n}-B_{k} B_{n+1} & =(-1)^{n} B_{k} B_{n-k} \\
B_{k-1} B_{n}-B_{k-1} B_{n+1} & =(-1)^{n} B_{k-1} B_{n-k+1}
\end{aligned}
$$

Adding equation (5.4) and (5.5), we get

$$
\begin{aligned}
& B_{k} B_{n}+B_{k-1} B_{n}-B_{k} B_{k+1}-B_{k-1} B_{n+1} \\
& =(-1)^{n} B_{k} B_{n-k}+(-1)^{n} B_{n-k+1} \\
& \left(B_{k}+B_{k-1}\right) B_{n}-\left(B_{k}+B_{k-1}\right) B_{n+1} \\
& =(-1)^{n}\left(B_{k} B_{n-k}+B_{n-k+1}\right) \\
& B_{k+1} B_{n}-B_{k+1} B_{n+1}=(-1)^{n} B_{k+1} B_{n-k-1}
\end{aligned}
$$

Which is precisely our identity when $\mathrm{k}=\mathrm{m}$

Hence $B_{m+1} B_{n}-B_{m+2} B_{n+1}=(-1)^{n} B_{m+1} B_{n-m-1}, n \geq 1$.

Theorem (5.4). For every positive integer n, prove that

$$
B_{2 n}=B_{2 n+1}-B_{2 n-1}
$$

Proof: we shall have proved this identity by induction matched over $n$.

$$
\begin{aligned}
& \text { For } n=0, B_{2 \times 0}=B_{2 \times 0+1}-B_{2 \times 0-1} \\
& B_{0}=B_{1}-B_{-1} \\
& 2 s=1+s-(1+s-2 s) \\
& 2 s=1+s-(1-s) \\
& 2 s=2 s
\end{aligned}
$$

which is also true for $\mathrm{n}=0$.

When $\mathrm{n}=1$ than

$$
\begin{aligned}
& B_{2 \times 1}=B_{2 \times 1+1}-B_{2 \times 1-1} \\
& B_{2}=B_{3}-B_{1} \\
& (1+3 s)=2+4 s-(1+s) \\
& (1+3 s)=1+3 s
\end{aligned}
$$


which is also true for $\mathrm{n}=1$.

For $\mathrm{n}=\mathrm{k}$

$$
B_{2 k}=B_{2 k+1}-B_{2 k}
$$

For $\mathrm{n}=\mathrm{k}$ which is also true.

Now assume that identity is true for $n=1,2,3 \ldots \ldots . . k$ and

We so that it holds:

For $\mathrm{n}=\mathrm{k}+1$, then by assumption

$$
\begin{aligned}
B_{2(k+1)} & =B_{2(k+1)+1}-B_{2(k+1)-1} \\
B_{2 k+2}= & B_{2 k+3}+B_{2 k+1} \\
= & \left(B_{2 k+2}+B_{2 k+1}\right)-B_{2 k+1} \\
= & B_{2 k+2}
\end{aligned}
$$

Which is also true, for $\mathrm{n}=\mathrm{k}+1$

Hence, the result is true for all.

Theorem (5.5). For every positive integer n, prove that

$$
(1+s) F_{n-1}=B_{n}-B_{n-2}, n \geq 2 .
$$

Proof: we shall Prove this identity by induction over $n$, for $n=2$

$$
\begin{aligned}
& (1+s) F_{n-2}=(1+s)=(1+s) F_{1} \\
& =(1+s) .1 \\
& =1+s \\
& =B_{2}-B_{0}
\end{aligned}
$$

Now suppose that identity hold for $\mathrm{n}=\mathrm{k}-1, \mathrm{n}=\mathrm{k}-2$ than,

$$
\begin{aligned}
& (1+s) F_{k-2}=B_{k-1}-B_{k-3} \\
& (1+s) F_{k-3}=B_{k-2}-B_{k-4}
\end{aligned}
$$

On adding equation (5.8) \& (5.9) we get,

$$
\begin{aligned}
& (1+s) F_{k-2}+(1+s) B_{k-3} \\
& =\left(B_{k-1}+B_{k-2}\right)-\left(B_{k-3}+B_{k-4}\right) \\
& (1+s)\left(F_{k-2}+B_{k-3}\right)=B_{k}-B_{k-2} \\
& (1+s) F_{k-1}=B_{k}-B_{k-2}
\end{aligned}
$$

which is true for $\mathrm{n}=\mathrm{k}$,

$$
(1+s) F_{n-1}=B_{n}-B_{n-2}, n \geq 2 .
$$

Theorem(5. 6). For every positive integer n,

$$
B_{3}+B_{6}+B_{9}+\ldots \ldots .+B_{3 n}=\frac{1}{2}\left[B_{3 n+2}-(1+3 s)\right]
$$

Proof. By using Binet's formula, we have

$$
\begin{aligned}
& B_{3}+B_{6}+B_{9}+\ldots \ldots+B_{3 n} \\
& =\frac{\mathfrak{R}_{1}^{3}-\mathfrak{R}_{2}^{3}}{\sqrt{5}}+s\left(\mathfrak{R}_{1}^{3}+\mathfrak{R}_{2}^{3}\right)+\frac{\mathfrak{R}_{1}^{6}-\mathfrak{R}_{2}^{6}}{\sqrt{5}} \\
& +s\left(\mathfrak{R}_{1}^{6}+\mathfrak{R}_{2}^{6}\right)+\ldots . .+\frac{\mathfrak{R}_{1}^{3 n}-\mathfrak{R}_{2}^{3 n}}{\sqrt{5}}+s\left(\mathfrak{R}_{1}^{3 n}+\mathfrak{R}_{2}^{3 n}\right) \\
& =\frac{1}{\sqrt{5}}\left[\begin{array}{c}
\left(\mathfrak{R}_{1}^{3}+\mathfrak{R}_{1}^{6}+\mathfrak{R}_{1}^{9} \ldots \mathfrak{R}_{1}^{3 n}\right) \\
-\left(\mathfrak{R}_{2}^{3}+\mathfrak{R}_{2}^{6}+\mathfrak{R}_{2}^{9} \ldots . \mathfrak{R}_{2}^{3 n}\right)
\end{array}\right]
\end{aligned}
$$

$$
\begin{aligned}
& +s\left[\left(\mathfrak{R}_{1}^{3}+\mathfrak{R}_{1}^{6}+\mathfrak{R}_{1}^{9} \ldots . \mathfrak{R}_{1}^{3 n}\right)\right] \\
& =\frac{1}{\sqrt{5}}\left[\left(\frac{\left.\left.\mathfrak{R}_{2}^{3}+\mathfrak{R}_{2}^{6}+\mathfrak{R}_{2}^{9} \ldots \mathfrak{R}_{2}^{3 n}\right)\right]}{\mathfrak{R}_{1}^{3}-1}\right)-\left(\frac{\mathfrak{R}_{2}^{3 n+3}-\mathfrak{R}_{2}^{3}}{\mathfrak{R}_{2}^{3}-1}\right)\right] \\
& +s\left[\frac{\mathfrak{R}_{1}^{3 n+3}-\mathfrak{R}_{1}^{3}}{\mathfrak{R}_{1}^{3}-1}+\frac{\mathfrak{R}_{2}^{3 n+3}-\mathfrak{R}_{2}^{3}}{\mathfrak{R}_{2}^{3}-1}\right] \\
& =\frac{1}{\sqrt{5}}\left[\frac{\mathfrak{R}_{1}^{3 n+2}-\mathfrak{R}_{1}^{2}}{2}-\left(\frac{\mathfrak{R}_{2}^{3 n+2}-\mathfrak{R}_{2}^{2}}{2}\right)\right] \\
& +s\left[\frac{\mathfrak{R}_{1}^{3 n+2}-\mathfrak{R}_{1}^{2}}{2}+\frac{\mathfrak{R}_{2}^{3 n+2}-\mathfrak{R}_{2}^{2}}{2}\right] \\
& =\frac{1}{2}\left[\frac{\mathfrak{R}_{1}^{3 n+2}-\mathfrak{R}_{2}^{3 n+2}}{\sqrt{5}}+s\left(\mathfrak{R}_{1}^{3 n+2}+\mathfrak{R}_{2}^{3 n+2}\right)\right] \\
& =\frac{1}{2}\left(B_{3 n+2}-B_{2}\right) \\
& =\frac{1}{2}\left[\frac{\mathfrak{R}_{1}^{2}-\mathfrak{R}_{2}^{2}}{\left.\sqrt{5}+s\left(\mathfrak{R}_{1}^{2}+\mathfrak{R}_{2}^{2}\right)\right]}\right. \\
& =
\end{aligned}
$$

This is completes the proof.

Theorem (5.7). For every positive integer

$$
\begin{aligned}
& B_{5}+B_{8}+B_{11}+\ldots \ldots+B_{3 n+2} \\
& =\frac{1}{2}\left[B_{3 n+4}-3-7 s\right]
\end{aligned}
$$

Proof. By using Binet's formula, we have

$$
\begin{aligned}
& B_{5}+B_{8}+B_{11}+\ldots \ldots+B_{3 n+2} \\
& =\frac{\mathfrak{R}_{1}^{5}-\mathfrak{R}_{2}^{5}}{\sqrt{5}}+s\left(\mathfrak{R}_{1}^{5}+\mathfrak{R}_{2}^{5}\right)+\frac{\mathfrak{R}_{1}^{8}-\mathfrak{R}_{2}^{8}}{\sqrt{5}} \\
& +s\left(\mathfrak{R}_{1}^{8}+\mathfrak{R}_{2}^{8}\right)+\ldots .+\frac{\mathfrak{R}_{1}^{3 n+2}-\mathfrak{R}_{2}^{3 n+2}}{\sqrt{5}} \\
& +s\left(\mathfrak{R}_{1}^{3 n+2}+\mathfrak{R}_{2}^{3 n+2}\right) \\
& =\frac{1}{\sqrt{5}}\left[\begin{array}{c}
\left(\mathfrak{R}_{1}^{5}+\mathfrak{R}_{1}^{8}+\mathfrak{R}_{1}^{11} \ldots . \mathfrak{R}_{1}^{3 n+2}\right) \\
-\left(\mathfrak{R}_{2}^{5}+\mathfrak{R}_{2}^{8}+\mathfrak{R}_{2}^{11} \ldots . \mathfrak{R}_{2}^{3 n+2}\right)
\end{array}\right] \\
& +s\left[\begin{array}{l}
\left(\mathfrak{R}_{1}^{5}+\mathfrak{R}_{1}^{8}+\mathfrak{R}_{1}^{11} \ldots . \mathfrak{R}_{1}^{3 n+2}\right) \\
-\left(\mathfrak{R}_{2}^{5}+\mathfrak{R}_{2}^{8}+\mathfrak{R}_{2}^{11} \ldots . \mathfrak{R}_{2}^{3 n+2}\right)
\end{array}\right] \\
& =\frac{1}{\sqrt{5}}\left[\left(\frac{\mathfrak{R}_{1}^{3 n+5}-\mathfrak{R}_{1}^{5}}{\mathfrak{R}_{1}^{5}-1}\right)-\left(\frac{\mathfrak{R}_{2}^{3 n+5}-\mathfrak{R}_{2}^{5}}{\mathfrak{R}_{2}^{5}-1}\right)\right] \\
& +s\left[\frac{\mathfrak{R}_{1}^{3 n+5}-\mathfrak{R}_{1}^{3}}{\mathfrak{R}_{1}^{5}-1}+\frac{\mathfrak{R}_{2}^{3 n+5}-\mathfrak{R}_{2}^{5}}{\mathfrak{R}_{2}^{5}-1}\right]
\end{aligned}
$$




$$
\begin{aligned}
& =\frac{1}{\sqrt{5}}\left[\frac{\mathfrak{R}_{1}^{3 n+4}-\mathfrak{R}_{1}^{4}}{2}-\left(\frac{\mathfrak{R}_{2}^{3 n+4}-\mathfrak{R}_{2}^{4}}{2}\right)\right] \\
& +s\left[\frac{\mathfrak{R}_{1}^{3 n+4}-\mathfrak{R}_{1}^{4}}{2}+\frac{\mathfrak{R}_{2}^{3 n+4}-\mathfrak{R}_{2}^{4}}{2}\right] \\
& =\frac{1}{2}\left[\frac{\mathfrak{R}_{1}^{3 n+4}-\mathfrak{R}_{2}^{3 n+4}}{\sqrt{5}}+s\left(\mathfrak{R}_{1}^{3 n+4}+\mathfrak{R}_{2}^{3 n+4}\right)\right] \\
& -\frac{1}{2}\left[\frac{\mathfrak{R}_{1}^{4}-\mathfrak{R}_{2}^{4}}{\left.\sqrt{5}+s\left(\mathfrak{R}_{1}^{4}+\mathfrak{R}_{2}^{4}\right)\right]}\right. \\
& =\frac{\left(B_{3 n+4}-B_{4}\right)}{2} \\
& =\frac{1}{2}\left[B_{3 n+4}-3-7 s\right]
\end{aligned}
$$

This is completes the proof.

Theorem (5.8). For positive integer $n$, prove that

$$
B_{n+1} B_{n-1}-B_{n}^{2}=(-1)^{n+1}\left(5 s^{2}-1\right), n \geq 1
$$

This can be derived same as theorem (1.4)

Theorem (5.9). For positive integer n, prove that

$$
B_{n}^{2}=(-1)^{n+1}(1+s) B_{n}, n \geq 1
$$

This can be derived same as theorem (1.4).

Theorem (5.10). For every integer $n \geq 0$, prove that

$$
B_{2 n}=F_{2 n}+s L_{2 n}, n \geq 0
$$

This can be derived same as theorem (1.4)

Theorem (5.11). For every integer $n \geq 0$, prove that

$$
F_{n+1}+s L_{n+1}=B_{n+1}, n \geq 0
$$

This can be derived same as theorem (1.4).

Theorem (5.12). For every integer $n \geq 1$, prove that

$$
(1+s) F_{n}+2 s F_{n-1}=B_{n+1}-B_{n-1}, n \geq 1
$$

This can be derived same as theorem (1.4).

\section{Connection Formulae}

In this section, connection formulae of Generalized Fibonacci-Like sequence associated with Fibonacci and Lucas sequences, induction method are presented.

Theorem (6.1). For positive integer n, Prove that

$$
2 s F_{n-1}=B_{n-1}-B_{n-2}, n \geq 3
$$

Proof: We shall prove this identity by induction. It is easy to show that for $\mathrm{n}=3$

$$
\begin{aligned}
2 s F_{n-1}=2 s F_{3-1} & =2 s F_{2} \\
& =2 s F_{2} \\
& =2 s .1=2 s \\
& =B_{2}-B_{1} .
\end{aligned}
$$

Now suppose the identity holds $\mathrm{n}=\mathrm{k}-1, \mathrm{n}=\mathrm{k}-2$. Then,

$$
\begin{aligned}
& 2 s F_{k-2}=B_{k-2}-B_{k-3} . \\
& 2 s F_{k-3}=B_{k-3}-B_{k-4} .
\end{aligned}
$$

On adding equation (6.2) and (6.3), we get

$$
\begin{aligned}
& \text { i.e. } 2 s F_{k-2}+2 s F_{k-3}=\left(B_{k-2}+B_{k-3}\right)-\left(B_{k-3}+B_{k-4}\right) \\
& 2 s\left(F_{k-2}+F_{k-3}\right)=B_{k-1}-B_{k-2} \\
& 2 s F_{k-1}=B_{k-1}-B_{k-2}
\end{aligned}
$$

Which is precisely our identity when $\mathrm{n}=\mathrm{k}$.

Hence $2 \mathrm{~s} \mathrm{~F}_{\mathrm{n}-1}=\mathrm{B}_{\mathrm{n}-1}-\mathrm{B}_{\mathrm{n}-2, \mathrm{n}} \geq 3$.

Theorem (6.2). For positive integer n, Prove that

$$
2 s L_{n-1}=B_{n}-B_{n-1}, n \geq 2
$$

Proof: We shall Prove this identity by induction over $n$ for $\mathrm{n}=2$

$$
\begin{aligned}
2 s L_{n-1}=2 b L_{2-1} & =2 s L_{1} \\
& =2 s .1=2 s=B_{2}-B_{1} .
\end{aligned}
$$

Now suppose the identity holds for $\mathrm{n}=\mathrm{k}-1, \mathrm{n}=\mathrm{k}-2$. Then,

$$
\begin{aligned}
& 2 s L_{k-2}=B_{k-1}-B_{k-2} \\
& 2 s L_{k-3}=B_{k-2}-B_{k-3}
\end{aligned}
$$

Adding equation (6.5) and (6.6), we get

$$
\begin{aligned}
& \text { i.e. } 2 s\left(L_{k-2}+L_{k-3}\right)=\left(B_{k-1}+B_{k-2}\right)-\left(B_{k-2}+B_{k-3}\right) \\
& 2 s L_{k-1}=B_{k}-B_{k-1}
\end{aligned}
$$

Which is true for $\mathrm{n}=\mathrm{k}$,

Hence $2 \mathrm{~s} \mathrm{~L}_{\mathrm{n}-1}=\mathrm{B}_{\mathrm{n}}-\mathrm{B}_{\mathrm{n}-1, \mathrm{n}} \geq 2$.

Theorem (6.3). For positive integer $n$, prove that

$$
(1+s) L_{n-1}=B_{n-1}-F_{n-2}, n \geq 2
$$

Theorem (6.4). For positive integer $n$, prove that

$$
(1+s) L_{n-1}=B_{n}-B_{n-2}, n \geq 2
$$

Theorem (6.5). For positive integer $n$, prove that

$$
B_{n-3}=2 s L_{n-2}+F_{n-3}, n \geq 3 \text {. }
$$

Theorem (6.6). For positive integer $n$, prove that

$$
2 s F_{n-1}=B_{n+1}-2 B_{n-1}, n \geq 2 \text {. }
$$

\section{Some Determinant Identities}

There is a long tradition of using matrices and determinants to study Fibonacci numbers. Problems on determinants of Fibonacci sequence and Lucas sequence are appeared in various issues of Fibonacci Quarterly. T. Koshy [15] explained two chapters on the use of matrices and determinants. Many determinant identities of generalized Fibonacci sequence are discussed in $[4,6]$ and [11]. In this section some determinant identities of Generalized Fibonacci-Like sequence are presented. Entries of determinants are satisfying the recurrence relation of Generalized Fibonacci-Like sequence and other sequences.

Theorem (7.1). Let $\mathrm{n}$ be a positive integer. Then

$$
\left|\begin{array}{ccc}
\mathrm{B}_{\mathrm{n}} & \mathrm{F}_{\mathrm{n}} & 1 \\
\mathrm{~B}_{\mathrm{n}+1} & \mathrm{~F}_{\mathrm{n}+1} & 1 \\
\mathrm{~B}_{\mathrm{n}+2} & \mathrm{~F}_{\mathrm{n}+2} & 1
\end{array}\right|=\left[F_{n} B_{n+1}-B_{n} F_{n+1}\right]
$$


Proof: Let

$$
\Delta=\left|\begin{array}{ccc}
B_{n} & F_{n} & 1 \\
B_{n+1} & F_{n+1} & 1 \\
B_{n+2} & F_{n+2} & 1
\end{array}\right|
$$

And

$$
\begin{gathered}
\text { assume } \mathrm{B}_{\mathrm{n}}=\mathrm{a}, \mathrm{B}_{\mathrm{n}+1}=\mathrm{b}, B_{\mathrm{n}+2}=\mathrm{a}+\mathrm{b} \\
F_{n}=P, F_{n+1}=q, F_{n+2}=p+q
\end{gathered}
$$

Now substituting the value of equation (7.2) \& (7.3) in (7.1), we get

$$
\Delta=\left|\begin{array}{ccc}
a & p & 1 \\
b & q & 1 \\
a+b & p+q & 1
\end{array}\right|
$$

Applying $R_{1} \rightarrow R_{1}-R_{2}$

$$
\Delta=\left|\begin{array}{ccc}
a-b & p-q & 0 \\
b & q & 1 \\
a+b & p+q & 1
\end{array}\right|
$$

Applying $\mathrm{R}_{2} \rightarrow R_{2}-R_{3}$

$$
\begin{gathered}
\Delta=\left|\begin{array}{ccc}
a-b & p-q & 0 \\
b-(a+b) & q-(p+q) & 0 \\
a+b & p+q & 1
\end{array}\right| \\
\Delta=\left|\begin{array}{ccc}
a-b & p-q & 0 \\
-a & -p & 0 \\
a+b & p+q & 1
\end{array}\right| \\
\Delta=[p b-a q]
\end{gathered}
$$

Again substituting the values of the equation (7.2) and (7.3) in (7.4).

$$
\begin{aligned}
& \text { We get } \Delta=\left[F_{n} B_{n+1}-B_{n} F_{n+1}\right] . \\
& \text { Hence }\left|\begin{array}{ccc}
B_{n} & F_{n} & 1 \\
B_{n+1} & F_{n+1} & 1 \\
B_{n+2} & F_{n+2} & 1
\end{array}\right|=\left[F_{n} B_{n+1}-B_{n} F_{n+1}\right] \text {. }
\end{aligned}
$$

Similarly we can derive following identities:

Theorem (7.2). For every integer $n \geq 2$, prove that

$$
\left|\begin{array}{ccc}
B_{n} & B_{n+1} & B_{n+2} \\
B_{n+2} & B_{n} & B_{n+1} \\
B_{n+1} & B_{n+2} & B_{n}
\end{array}\right|=2\left(B_{n}^{3}+B_{n+1}^{3}\right)
$$

Theorem (7.3). For any integer $n \geq 0$, prove that

$$
\left|\begin{array}{ccc}
B_{n} & L_{n} & 1 \\
B_{n+1} & L_{n+1} & 1 \\
B_{n+2} & L_{n+2} & 1
\end{array}\right|=2\left(L_{n} B_{n+1}-B_{n} L_{n+1}\right)
$$

Theorem (7.4). For every positive integer n, prove that

$$
\left|\begin{array}{ccc}
B_{n}+B_{n+1} & B_{n+1}+B_{n+2} & B_{n+2}+B_{n} \\
B_{n+2} & B_{n} & B_{n+1} \\
1 & 1 & 1
\end{array}\right|=0
$$

Theorem (7.5). For every positive integer n, prove that

$$
\left|\begin{array}{ccc}
1+B_{n} & B_{n+1} & B_{n+2} \\
B_{n} & 1+B_{n+1} & B_{n+2} \\
B_{n} & B_{n+1} & 1+B_{n+2}
\end{array}\right|=1+B_{n}+B_{n+1}+B_{n+2}(7.8)
$$

The identities from (7.1) to (7.4) can be proved similarly.

\section{Conclusions}

In this paper, Generalized Fibonacci-Like sequence is introduced. Some standard identities of generalized Fibonacci-Like sequence associated with Fibonacci and Lucas sequences have been obtained and derived using Binet's formula. Also some determinant identities have been established and derived.

\section{Acknowledgement}

We would like to thank the anonymous referees for numerous helpful suggestions.

\section{References}

[1] A. F. Horadam: A Generalized Fibonacci Sequence, American Mathematical Monthly, Vol. 68. (5), 1961, 455-459.

[2] A. F. Horadam: Basic Properties of a Certain Generalized Sequence of Numbers, The Fibonacci Quarterly, Vol. 3 (3), 1965, 161-176.

[3] A.T. Benjamin and D. Walton, Counting on Chebyshev polynomials, Math. Mag. 82, 2009, 117-126.

[4] B. Singh, O. Sikhwal and S. Bhatnagar: Fibonacci-Like Sequence and its Properties, Int. J. Contemp. Math. Sciences, Vol. 5 (18), 2010, 859-868.

[5] B. Singh, Omprakash Sikhwal, and Yogesh Kumar Gupta, "Generalized Fibonacci-Lucas Sequence, Turkish Journal of Analysis and Number Theory, Vol.2, No.6. (2014), 193-197.

[6] B. Singh, S. Bhatnagar and O. Sikhwal: Fibonacci-Like Sequence, International Journal of Advanced Mathematical Sciences, 1 (3) (2013) 145-151.

[7] D. V. Jaiswal: On a Generalized Fibonacci sequence, Labdev J. Sci. Tech. Part A 7, 1969, 67-71.

[8] M. Edson and O. Yayenie: A New Generalization of Fibonacci sequence and Extended Binet's Formula, Integers Vol. 9, 2009, 639-654.

[9] M. E. Waddill and L. Sacks: Another Generalized Fibonacci sequence, The Fibonacci Quarterly, Vol. 5 (3), 1967, 209-222.

[10] M. Singh, Y. Gupta, O. Sikhwal, Generalized Fibonacci-Lucas Sequences its Properties, Global Journal of Mathematical Analysis, 2 (3) 2014, 160-168.

[11] M. Singh, Y. Gupta, O. Sikhwal, "Identities of Generalized Fibonacci-Like Sequence." Turkish Journal of Analysis and Number Theory, vol.2, no. 5 (2014): 170-175. doi:10.12691/tjant 2-5-3.

[12] S. Falcon and A. Plaza: On the Fibonacci $K$ - Numbers, Chaos, Solutions \& Fractals, Vol. 32 (5), 2007, 1615-1624.

[13] Singh, M., Sikhwal, O., and Gupta, Y., Generalized FibonacciLucas Polynomials, International Journal of Advanced Mathematical Sciences, 2 (1) (2014), 81-87

[14] S. Vajda, Fibonacci \& Lucas Numbers, and the Golden Section, Theory and Applications, Ellis Horwood Ltd., Chichester, 1989.

[15] T. Koshy, Fibonacci and Lucas Numbers with Applications, Wiley-Interscience Publication, New York (2001).

[16] Y. Gupta, M. Singh, and O. Sikhwal, Generalized Fibonacci-Like Polynomials and Some Identities Global Journal of Mathematical Analysis, 2 (4) (2014) 249-258. 